\title{
Integração ensino-serviço: as experiências e fragilidades na atenção básica
}

\author{
Teaching-Service integration: Experiences and fragilities in primary care
}

Integración enseñanza-servicio: experiencias y fragilidades en la atención primaria

Deborah Holanda da Silva Brayde ${ }^{1 *}$, José Antônio Cordero da Silva", Santino Carvalho Franco.

\section{RESUMO}

Objetivo: Analisar as experiências e opiniões dos docentes da atenção básica, em relação à prática de integração ensino-serviço. Métodos: Trata-se de uma pesquisa qualitativa e descritiva, na Universidade do Estado do Pará (UEPA), com docentes do módulo de Gestão, integração, ensino e comunidade (GIESC), através de entrevista com 15 perguntas e utilizando-se a técnica de análise de conteúdo. Resultados: Os resultados obtidos estavam relacionados a ausência de infraestrutura física e segurança nos locais de prática, falta de integração das atividades de ensino e assistência e ausência de maior capacitação dos docentes e aplicação de atividades à realidade da comunidade. As atividades do GIESC deveriam ser mais integradas a assistência nas Unidades de saúde; a instituição de ensino deveria ser mais participativa no sentido de desenvolver mecanismos de melhorias aos locais de prática, como também na formação docente, o retorno à comunidade deveria ter mais enfoque de intervenção e não apenas diagnóstico da realidade vivenciada. Conclusão: A metodologia de integração ensino-serviço é uma boa estratégia de formação médica, permitindo 0 alcance do perfil do egresso sugerido pelas diretrizes, mas que precisa ser melhor adequada dentro da UEPA, através de melhor planejamento das atividades e maior participação da Universidade nesse processo.

Palavras-chave: Serviços de integração docente-assistencial, Educação médica, Atenção básica.

\begin{abstract}
Objective: Analyze the experiences and opinions of primary care of teachers, regarding the practice of teaching-service integration. Methods: This is a qualitative and descriptive research carried out at the University of Pará (UEPA), with teachers from the Management, Integration, Teaching and Community module (GIESC), through an interview with 15 questions using the technique of the content analysis. Results: The results obtained were relate to the lack of physical infrastructure and safety in the places of practice, lack of integration of teaching and assistance activities and absence of greater training of teachers and application of activities to the reality of the community. The activities of the GIESC should be more integrated to the assistance in the Health Units; the educational institution should be more participatory in developing mechanisms for improving places of practice and also in teacher training, the return to the community should be more focused on intervention and not just a diagnosis of reality. Conclusion: The teaching-service integration methodology is a good medical training strategy, allowing the achievement of the egress profile suggested by the guidelines, but which needs to be better adapted within UEPA, through better planning of activities and greater participation of the University in this process.
\end{abstract}

Key words: Teaching care integration services, Medical education, Primary care.

1Universidade do Estado do Pará (UEPA),Belém-PA. `E-mail: deborah_brayde@yahoo.com.br

SUBMETIDO EM: 4/2019

ACEITO EM: 5/2019

PUBLICADO EM: 7/2019

REAS/EJCH | Vol. Sup. 26| e757 | DOI: https://doi.org/10.25248/reas.e757.2019 Página 1 de 9 


\section{RESUMEN}

Objetivo: Analizar las experiencias y opiniones de los docentes de atención primaria en relación con la práctica de la integración enseñanza-servicio. Métodos: Se trata de un estudio cualitativo y descriptivo en la Universidad del estado de Pará (UEPA), con profesores de la gerencia, integración, docencia y módulo comunitario (GIESC), a través de entrevistas con 15 preguntas y utilizando la técnica de análisis de contenidos. Resultados: Los resultados obtenidos estuvieron relacionados con la ausencia de infraestructura física y seguridad en los lugares de práctica, la falta de integración de las actividades docentes y asistenciales y la ausencia de una mayor formación de docentes y la aplicación de actividades a La realidad comunitaria. Las actividades del GIESC deberían estar más integradas para la atención en las unidades sanitarias; La institución educativa debe ser más participativa para desarrollar mecanismos de mejora a los lugares de práctica, así como en la educación del profesorado, el retorno a la comunidad debe centrarse más en la intervención y no sólo en el diagnóstico de la realidad experimentado. Conclusión: La metodología de integración enseñanza-servicio es una buena estrategia de formación médica, permitiendo el logro del perfil de la salida sugerida por las directrices, pero que debe ser mejor adaptada dentro de la UEPA, a través de una mejor planificación de las actividades y Mayor participación de la Universidad en este proceso.

Palabras clave: Servicios de integración docente-asistencial, Educación médica, Atención primaria.

\section{INTRODUÇÃO}

Mudanças no cenário internacional, motivadas pelo relatório Lalonde e a Conferência de Alma-Ata, influenciaram o movimento sanitarista brasileiro, na década de 80 , levando a criação da Lei Orgânica da Saúde (8.080) e do Sistema Único de Saúde (SUS). Este trouxe princípios relacionados a universalidade, integralidade e equidade, influenciando a formação de recursos humanos em saúde (TEMPSK $P$ e BORBA $M, 2009$ ).

Essa transição do modelo mecanicista e focado em princípios diagnósticos e de terapia medicamentosa, para o modelo biopsicossocial, que juntamente com o surgimento do SUS e do programa Saúde da Família, reorganizaram os serviços de saúde, demonstrando uma deficiência na formação médica, que não atendia as necessidades da população, não proporcionando aos discentes vivenciarem a atenção primária no contexto do usuário e das equipes de saúde (SOUZA CFT, et al., 2013).

O ensino médico voltado a uma assistência individual e curativa incorporou mudanças que atendessem esses novos princípios, refletidas nas Diretrizes Curriculares Nacionais (DCN) desde 2001, que entre outras coisas trouxe a necessidade de ampliação e diversificação dos cenários de prática, desde o início da formação, principalmente na atenção básica, permitindo a interação com as equipes multiprofissionais, priorizando as heterogeneidades sociais e as diferentes condições de saúde, ampliando assim a formação profissional, desenvolvendo a autonomia e a capacidade decisória dos discentes e utilizando a integração ensino-serviço como vínculo entre a formação médica e as necessidades sociais de saúde (AZEVEDO CC, 2013; BRANDÃO ERM, et al., 2013; MARANHÃO EA, 2001).

As últimas DCN 2014 estabelecem ainda como perfil do egresso ser generalista, humanista, crítico, reflexivo e ético, que possa atuar nos três níveis de atenção à saúde, considerando a integralidade da assistência, de forma individual e coletiva, valorizando os aspectos sociais do processo saúde-doença (BRASIL, 2014). A integração ensino-serviço surge como instrumento de ensino-aprendizagem, permitindo ao discente vivenciar a realidade do SUS e seus princípios, integrando teoria e prática com multidisciplinaridade, desenvolvendo a proatividade e a autonomia do aluno (PIZINATTO A, et al., 2012).

Essa estratégia que procura facilitar o processo de formação em saúde caracteriza-se como um processo firmado entre estudantes, docentes, gestores e profissionais da assistência, cujo objetivo é promover a melhoria dos serviços prestados, gerando uma formação profissional de qualidade, satisfação e capacitação dos funcionários. Portanto, é um reposicionamento da formação em saúde, gerando transformações na 
aquisição do conhecimento e na assistência prestada à população (BATISTA SHSS, et al., 2015; PIZINATTO A, et al., 2012).

Essa interação da teoria com a prática pode propiciar ao discente maior compromisso com a população atendida, tornando-o um profissional mais humano e reflexivo. Por isso entende-se que esta prática é bastante significativa na formação médica, permitindo-se alcançar os objetivos estabelecidos nas DCN, mas os seus resultados dependem de diversos fatores e não da mera observação da realidade: a infraestrutura dos ambientes, a participação das instituições de ensino, o comprometimento do docente/preceptor, o interesse do aluno, a participação das equipes de saúde, correlação entre teoria e pratica e a problematização das situações, são fundamentais para o êxito dessa estratégia de ensino (MARSDEN M, 2009).

A Universidade do Estado do Pará, buscando adequar-se as novas diretrizes do ensino médico estabeleceu a problematização na atenção básica através do eixo Gestão, Integração, Ensino, Serviço e Comunidade (GIESC), que desenvolve-se nos quatro primeiros anos do curso, através de 8 sub módulos, com duração de 20 semanas e carga-horária semanal de 4 horas, permitindo ao aluno desenvolver habilidades e atitudes necessárias para atuar na estratégia Saúde da Família, participando ativamente em programas de base comunitária, com abordagem centrada na pessoa e com princípios e ferramentas da atenção primária (PRESTES EX, et al., 2015).

Diante disso, este trabalho teve por objetivo, analisar as experiências e as opiniões dos docentes do GIESC, destacando-se as principais fragilidades destas atividades de ensino na atenção básica.

\section{MÉTODOS}

O presente estudo trata-se de uma pesquisa de abordagem, qualitativa e de caráter descritivo, ocorreu na Universidade Estado do Pará (UEPA), no curso de medicina, localizada na Travessa Perebebuí, № 2623, bairro do Marco, cidade de Belém, estado do Pará, no Campus II-CCBS (Centro de Ciências Biológicas e da Saúde).

Participaram do estudo, docentes do curso de Medicina do Campus em Belém, que desenvolvem suas atividades dentro do contexto da integração ensino-serviço, atuando na atenção primária, totalizando nove dos quinze docentes que atuam neste eixo.

Seguindo as orientações da resolução 466 de 12 de dezembro de 2012, a pesquisa foi submetida ao Comitê de Ética em Pesquisa (CEP) da UEPA, sendo aprovado pelo parecer 2.069.832, de 17 de maio de 2017.

A participação na pesquisa ocorreu voluntariamente, através do contato prévio com os docentes e o agendamento das entrevistas, que ocorreram no Campus II ou nas ESF em que os docentes atuam. Na ocasião os entrevistados foram informados sobre os objetivos da pesquisa e estes aceitaram participar mediante assinatura do Termo de Consentimento Livre e Esclarecido, em duas vias, sendo uma entregue ao participante.

Utilizou-se como instrumento de coleta de dados uma entrevista semiestruturada individual, com quinze perguntas, abordando questões da formação, prática docente e sobre as experiências e opiniões das atividades de integração ensino-serviço, na atenção primária à saúde.

Foi realizado um teste piloto para validação e adequação da entrevista, com três docentes de outra instituição de ensino privada, que utiliza a mesma metodologia de integração e serviço nos mesmos moldes do GIESC, resultando em adequações do instrumento.

Todas as entrevistas foram gravadas, com a autorização dos participantes, transcritas na íntegra e analisadas pela técnica de análise de conteúdo, elegendo-se cinco categorias: humanização do atendimento, contato com a realidade, dificuldades operacionais, organização e educação em saúde.

\section{RESULTADOS E DISCUSSÕES}

REAS/EJCH | Vol. Sup. 26| e757 | DOI: https://doi.org/10.25248/reas.e757.2019 Página $\mathbf{3}$ de $\mathbf{9}$ 
Foram entrevistados nove docentes, na sua totalidade médicos, com uma média de idade de 42,66 anos, a maioria do gênero feminino $(66,6 \%)$, com mais de dois anos de prática docente e $66,66 \%$ atuando em instituições públicas e privadas, a maioria com pós-graduação em atenção básica, sendo quadro com residência médica em Medicina de Família e Comunidade e cinco dos entrevistados com título de mestre.

Com base nos resultados obtidos e comparando com estudos semelhantes, observa-se discordância com Andrade SR, et al., (2015), que em seu estudo avaliava a percepção de preceptores de enfermagem sobre a parceria ensino-serviço na atenção básica, houve um predomínio de docentes femininos (100\%), idade entre 24 e 56 anos, com tempo de atuação docente de 26 anos.

\section{Categoria 1: Humanização do atendimento}

Sobre a mudança de perfil dos alunos, a grande diferença foi observada nas características de humanização dos discentes.

P1: "Eu acho que a postura deles já mudou muito, a gente tira por esses médicos novos, com uma nova forma de tratar o paciente, se relacionar com a equipe de saúde, responsabilidade com relação a promoção e a prevenção."

P2: "Boa evolução em relação ao contato com o paciente, também com uma visão humana, holística, que no currículo tradicional era mais distante. E também o fato do aluno conhecer a realidade do SUS em cenários reais de prática."

P3: "Alunos menos temerosos e com uma visão mais social."

P4: "Interesse maior pela atenção básica, pela integralidade da assistência"

P5: "Já mudou bastante, o que eu percebo, que o aluno já é mais crítico e teorizado, mas nos módulos iniciais são mais inseguros."

P6: "Maior compromisso, empenho, participação, iniciativa, sensibilidade e cuidados com as pessoas."

P7: "Com certeza. O aluno tem muito mais discernimento, iniciativa. Quando o aluno chega nas séries mais avançada: GIESC 7, 8 e no internato, ele é bem diferente do aluno do início.

P8: "Alunos mais motivados e humanizados."

P9: "Sensibilização maior, crescimento como pessoa e uma visão da integralidade da assistência."

Os docentes entendem que a integração ensino-serviço tem contribuído para mudança de perfil dos alunos, com uma visão de integralidade da assistência, com valorização da equipe multiprofissional, humanização e melhoria das relações interpessoais, refletindo na relação médico-paciente e na qualidade dos serviços prestados à comunidade, de acordo com Mardsen M (2009).

Marin MJS, et al. (2014) e Sousa MC, et al. (2016) verificaram que a mudança de cenários, gerou uma visão integral e humanizada dos discentes, a partir do dimensionamento da complexidade dos fatores que influenciam a saúde da população, aproximando estes dos princípios e diretrizes do SUS e até gerando influências na escolha de especialidades, como a opção pela Medicina de Família e Comunidade.

\section{Categoria 2: Contato com a realidade}

A integração ensino-serviço é fundamental para formação médica, porque prepara o discente para a realidade que ele vai viver, principalmente do sistema único de saúde, com suas precariedades e limitações.

Esta categoria demostra a importância do contato precoce com a realidade do sistema de saúde para a formação médica.

REAS/EJCH | Vol. Sup. 26| e757 | DOI: https://doi.org/10.25248/reas.e757.2019 Página 4 de 9 
P1: "Eu acho Fundamental, ou você vai formar médicos dentro de uma bolha e joga-los numa realidade que não é a que ele aprendeu, então eu acho que ele tem que aprender com a realidade e depois tentar mudar essa realidade."

P2: "De maneira geral é a questão de colocar o aluno de frente com a realidade do sus, em cenários reais, onde vai se deparar com questões não só de patologias do paciente, mas sociais e condições de trabalho."

P3: "Importância grande, porque na integração que você vai ter a simulação da realidade"

P4: "Extrema importância porque forma profissionais na realidade e dá retorno para a sociedade com esses profissionais."

P5: "Fundamental, muito importante, porque o aluno tem que ter o conhecimento científico e a habilidade para pôr em prática esse conhecimento em prática.

P6: "É bom para o aluno porque ele é inserido no campo de prática e aprende a Ihe dar com pessoas, com o sistema de saúde e como lhe dar com situações reais que os livros não vão ensinar. Também traz benefícios para a comunidade e para a equipe de saúde, pois sempre procura-se fazer a melhor prática e com melhor evidência, além de gerar um reforço a mais para equipe na assistência e na educação permanente. É bom para o docente, porque proporciona um ensino vinculado à realidade e não apenas dentro de condições ideais."

P7: "O aluno chega melhor preparado no internato e no mercado de trabalho."

"A entrada da academia no serviço, melhora a qualidade da assistência."

"Principalmente a experiência do contato com a realidade, o aluno que fica só na sala de aula ele não vê as falhas do sistema e não aprende a se sublimar. Na minha formação eu tive uma boa formação, mas só vi doente no internato. E hoje o aluno é inserido na realidade no primeiro semestre e recebe aquele impacto, ele se assusta, mas ganha experiência e vê como funciona a estrutura."

P8: "Importância de colocar o aluno na prática desde cedo, vivenciando a realidade da comunidade em que ele está inserido."

P9: "Faz com que o aluno entenda que ele será inserido em uma equipe e deverá aprender a trabalhar em equipe. $O$ aluno aprende desde a graduação a conviver, a ser mais resolutivo e a lhe dar com as adversidades do serviço."

Permitindo assim, que eles possam integrar teoria e prática, de uma maneira até resiliente, além de levar um retorno para a comunidade através das intervenções realizadas, como observado por Souza CFT, et al. (2013) que identificou através de sua pesquisa que a ausência de infraestrutura e materiais na ESF permitiu aos alunos conhecerem a realidade com que iriam se deparar no SUS e observar o esforço dos profissionais em garantir a assistência prestada, estimulando a formação de recursos humanos para a rede, como refere Pizzinato A, et al. (2012).

Já Ellery AEL, et al. (2013) relata que a integração permite a consolidação de conhecimentos e habilidades, tornando o profissional mais motivado e qualificado, com concepção preventiva e biopsicossocial, mais bem preparados para atuar na atenção básica e não apenas em consultórios particulares.

\section{Categoria 3: Dificuldades operacionais}

Quando abordados sobre as dificuldades de desenvolver as atividades de integração ensino-serviço na atenção básica, várias situações foram elencadas, mas as principais foram: A falta de infraestrutura: como a dificuldade de espaço físico para desenvolver as tarefas, com as unidades não preparadas para receber as atividades de ensino; A insegurança dos locais de prática, que como se desenvolvem em comunidades 
carentes geralmente encontra-se a ausência do poder público, dificultando a realização de visitas domiciliares e as ações na comunidade; A falta de integração das atividades de ensino com a programação da assistência: muitas vezes não se consegue adequar as programações do GIESC com a programação da unidade, prejudicando o cumprimento dos cronogramas e assim o aprendizado. Outro ponto é em relação ao retorno para comunidade que muitas vezes não é realizado pelo tempo curto do semestre ou mudanças de unidades e de docentes.

Nesta categoria os docentes foram questionados sobre as principais dificuldades operacionais no desenvolvimento das atividades de ensino-serviço.

P1: "A grande dificuldade é de dar feedback para a comunidade sobre as atividades e os trabalhos feitos, relacionada principalmente aos cronogramas das atividades."

P2: "Os alunos reclamam que muitas vezes não tem aonde fazer nada. Já aconteceu comigo, de chegar com os alunos e o administrativo me dizer que se quisesse atenderia lá fora, porque não tinha consultório para atender. Então tive que esperar um consultório vagar para atender."

P3: "Fui procurar uma unidade para dar aula e quase fui assaltado."

"Tive dificuldade de integrar o cronograma do GIESC com a necessidade da comunidade"

P4: "Adequar o perfil de atendimento com a necessidade dos alunos. Nem sempre o dia que você está na ESF, é o dia de atendimento do perfil de pacientes que você precisa."

"Dificuldade com espaço físico, muitas ESF's não estão adaptadas para receber os alunos e você depende da boa vontade da equipe."

P5: "Não tive dificuldades em realizar minhas atividades."

P6: "Maior dificuldade é em relação a demanda reprimida das unidades, a comunidade acha que é pronto-atendimento."

P7: "Já fui assaltado três vezes e soube de alunos que foram assaltados. As áreas são violentas e sem segurança."

"Na instituição privada criou-se uma escolta da polícia militar do estado, mas na estadual não tem."

P8: "Marco capacitação com ACS e eles não comparecem ou quando vou fazer visitas e não posso levar os alunos pela insegurança."

Corroborando com Pizzinato A, et al. (2012) e Baldoino AS e Veras RM (2016), em que as dificuldades operacionais estão na infraestrutura inadequada das unidades de saúde para receber o docente e o discente pelo espaço reduzido, a carência de profissionais em algumas equipes prejudicando a multidisciplinaridade e a dificuldade de inserir o professor e aluno em áreas de difícil acesso.

Para Costa NMSC, et al. (2012) o grande problema da inserção precoce no cenário de prática, estava nas questões estruturais da ESF e a insuficiente conversação entre a ESF e a Instituição de Ensino Superior (IES).

\section{Categoria 4: Organização}

As contribuições dos docentes para as melhorias das atividades do GIESC, estão relacionadas a três aspectos principais: Infraestrutura física: a sugestão estaria em firmar parcerias entre a Universidade e as secretárias Municipal de Saúde e estadual de segurança, para respectivamente adaptar as unidades para o ensino com espaço físico adequado e maior proteção policial nos locais de pratica (comunidades) que se 
desenvolvem em áreas de risco da cidade. $O$ item organização estaria relacionado as sugestões dos docentes para melhorias na prática de integração ensino-serviço:

P1:" Construir unidades próprias para o atendimento com um espaço reservado para o ensino"

P2: "Deveria ter uma parceria melhor das universidades com as prefeituras no sentido de prover um cenário de prática melhor, para o docente e o discente. $\mathrm{Na}$ questão da insegurança, a universidade que tem um poder de influência, poderia gerar parcerias para garantir a segurança dos alunos.

P3: "Quebrar o paradigma que o GIESC deve casar com as outras matérias do semestre. Ter uma liberdade maior de abordar os casos que aparecem na unidade, sem se prender ao cronograma."

P4: "Procurar a realidade daquela estratégia e então adequar a necessidade dos alunos ou que a equipe aceite liberar o perfil do paciente para o atendimento nas aulas."

P5: "Comunicação do pessoal da gestão, universidade e equipe da Estratégia. Uma orientação de como o docente deveria trabalhar nas comunidades. Um exemplo, foi quando eu fui fazer uma visita em uma unidade de referência, havia sido autorizado, mas quando cheguei com os alunos eles não sabiam da visita, sendo que foi fornecido o cronograma previamente."

P6: "Maior diálogo entre a equipe, docente e alunos e contemplar as necessidades da comunidade e do ensino."

P7: "Programação mais integrada com o serviço, muitas vezes temos que improvisar, porque quando a chega na unidade, é uma outra realidade. Falta uma integração de planejamento entre o ensino e o serviço"

P8: "Melhor integração da gestão com o ensino e o serviço, gerando melhor infraestrutura."

P9: "Infraestrutura melhor, motivar a equipe de saúde, melhorar a assistência dos programas de saúde, tudo isso pode melhorar as condições de trabalho e trazer um estímulo para equipe. Muitas vezes a universidade é colocada nas unidades e a equipe é obrigada a aceitar, mas será que não estamos sobrecarregando?"

Planejamento da disciplina: as atividades do GIESC deveriam ser mais integradas com as atividades assistenciais, desenvolvendo-se maior diálogo entre as equipes de saúde e os docentes para ajuste de cronogramas e atividades, para não prejudicar a formação do discente, uma vez que os eixos obrigatoriamente devem ser integrados nos diversos módulos, Retorno da IES para a comunidade e a para a equipe de saúde: a contrapartida da IES pode ser alcançada com benfeitorias de infraestrutura física para a unidade, capacitação e incentivo da equipe de saúde, com melhorias dos serviços prestados e intervenções a nível individual e coletivo para a comunidade. Albuquerque VS, et al. (2008), Demarzo MMP, et al. (2011) e Pizzinato A, et al. (2012) definem que tanto a estrutura quando as melhorias, bem como as atividades devem ser pactuações entre os diversos atores, a saber: as equipes, gestor local, comunidade e Instituição de Ensino, permitindo maiores adequações do serviço com as práticas e educacionais.

\section{Categoria 5: educação em saúde}

As atividades de intervenções na comunidade não foram desenvolvidas pelos professores entrevistados, principalmente pela dificuldade de tempo para realizar o retorno aos atendidos, devido os cronogramas curtos. Outro ponto estava relacionado aos trabalhos do final de semestre possuem uma abordagem mais teórica e diagnóstica da comunidade e não um perfil de intervenção, como propõe o método de Charles Marguerez explicitado por Prado ML, et al. (2012), com a aplicação prática à realidade, sendo a última etapa do ciclo, 
permitindo assim o alcance da coletividade. Na categoria 5, em que os docentes deveriam relatar suas experiências exitosas no GIESC, muitos docentes relataram situações pontuais sobre atendimentos individuais. Outras estavam relacionadas a atividades de educação em saúde, mas não houveram relados voltados para intervenção na comunidade.

P1: "Um trabalho sobre gravidez na adolescência, com pais e os alunos, com boa aceitação pela comunidade."

P2:" Uma Palestra de educação em saúde sobre alimentação nutrição e enteroparasitoses, quando passamos na mesma comunidade em outro semestre, vimos a população agindo, colocando em prática o que foi ensinado nas palestras.

P6:" Projeto de intervenção sobre peso e altura das crianças de uma creche, onde identificou-se problemas de crescimento, obesidade e desnutrição, gerando um diagnóstico para a equipe que pode intervir."

P8: "Campanha de capacitação sobre tuberculose para a comunidade, a partir dos vários casos de tuberculose atendidos e diagnosticados na comunidade."

Achado semelhante foi no estudo de Pizzinato A, et al. (2012) com um dos pontos críticos encontrados estava relacionado a necessidade de aumentar o número de intervenções realizadas pelos acadêmicos, inclusive integrando outras áreas do conhecimento, não ocorrendo devido a rotina do serviço, horários das aulas e dificuldades de alguns profissionais e docentes.

\section{CONCLUSÃO}

A integração ensino-serviço constitui-se em uma boa estratégia de ensino-aprendizagem na formação médica, porque aproxima o discente da realidade do sistema de saúde, principalmente o público, permitindo que se alcance o perfil do profissional exigido pelas diretrizes curriculares nacionais, como foi observado pelos docentes quando comparam a evolução dos alunos com decorrer dos semestres até o acesso ao internato. As dificuldades relacionadas a infraestrutura física e a insegurança dos locais de prática acadêmica foram os mais relevantes, seguidos da falta de apoio da gestão, pois acredita-se que a Universidade poderia contribuir mais com melhorias nas unidades, tanto física quanto de capacitação, impactando a assistência positivamente. O planejamento das atividades de ensino integrada a assistência, também poderia contribuir para um maior sucesso do processo de ensino-aprendizagem, adequando as necessidades dos módulos, que são ligados as atividades da tutoria e do morfofuncional, permitindo o alcance dos objetivos e maior aproveitamento do ensino.

\section{AGRADECIMENTOS E FINANCIAMENTO}

Agradecimentos à Universidade do Estado do Pará e ao Programa de Mestrado em Ensino em Saúde pela colaboração na realização desta pesquisa.

\section{REFERÊNCIAS}

1. ALBUQUERQUE VS, et al. A integração ensino-serviço no contexto dos processos de mudança na formação superior dos profissionais de saúde. Revista Brasileira de Educação Médica, 2008; 32(3): 356-362.

2. ANDRADE SR, et al. Percepções de enfermeiros docentes e assistenciais sobre a parceria ensino-serviço em unidades básicas de saúde. Interface: comunicação saúde educação, 2015; 19(54): 537-547.

3. AZEVEDO CC. Integração ensino-serviço em Unidades Básicas de Saúde do município de Maceió: preceptorias do PET-Saúde/ Saúde da Família na perspectiva da Ergologia e da Política Nacional de Humanização em Saúde. Tese (Doutorado em Saúde Pública) - Fundação Oswaldo Cruz. Escola Nacional de Saúde Pública Sérgio Arouca, Rio de Janeiro, 2013; 247p.

4. BALDOINO AS, VERAS RM. Análise das atividades de integração ensino-serviço desenvolvidas nos cursos de saúde da Universidade Federal da Bahia. Revista Brasileira de Enfermagem USP, 2016; 50: 017-024. 
5. BATISTA SHSS, et al. Formação em Saúde: reflexões a partir dos programas Pró-Saúde e PET-Saúde. Interface: comunicação saúde educação, 2015; 19: 743-752.

6. BRANDÃO ERM, et al. Práticas de integração ensino-serviço-comunidade: reorientando a formação médica. Revista Brasileira de Educação Médica, 2013; 37(4): 573-577.

7. BRASIL. Ministério da Educação. Resolução no 3, de 20 de junho de 2014. Diretrizes curriculares nacionais do curso de medicina. Brasília, Diário Oficial da União, 23 jun. 2014 - seção 1, p. 8-11.

8. COSTA NMSC, et al. Concepção sobre o bom professor de Medicina. Revista Brasileira de Educação Médica, 2012: 36(4): 499-504.

9. DEMARZO MMP, et al. Diretrizes para o ensino da Atenção Primária à Saúde na graduação em Medicina. Revista Brasileira de Medicina de Família e Comunidade, 2011; 6(19): 145-150.

10. ELLERY AEL, et al. Integração ensino, pesquisa e serviços em saúde: antecedentes, estratégias e iniciativas. Saúde e Sociedade, 2013; 22(1): 187-198.

11. MARANHÃO EA. Diretrizes curriculares nacionais dos cursos de graduação em Enfermagem, Medicina e Nutrição: relatório. Ministério da Educação. Conselho Nacional de Educação. Diário Oficial da União, Brasília, 23 outubro de 2001, seção 1E, p. 131.

12. MARIN MJS, et al. A integração ensino-serviço na formação de médicos e enfermeiros: a experiência da FAMEMA. Ciência \& Saúde Coletiva, 2014; 19(3): 967-974.

13. MARSDEN M. A indissociabilidade entre teoria e prática: experiências de ensino na formação de profissionais de saúde dos níveis superior e médio. Dissertação (Mestrado em Saúde Pública) - Fundação Oswaldo Cruz. Escola Nacional de Saúde Pública Sérgio Arouca. Rio de Janeiro, 2009; 140 p.

14. PIZINATTO A, et al. A integração ensino-serviço como estratégia na formação profissional para o SUS. Revista Brasileira de Educação Médica, 2012; 36(2): 170-177.

15. PRADO ML, et al. Arco de Charles Marguerez: refletindo estratégias de metodologia ativa na formação de profissionais de saúde. Escola Anna Nery, 2012; 16(1): 172-177.

16. PRESTES EX, et al. Projeto Político Pedagógico o Curso de Medicina da Universidade do Estado do Pará (UEPA) no Município de Belém - Pará. Belém: Universidade do Estado do Pará, 2015; 312p.

17. SOUZA CFT, et al. A atenção primária na formação médica: a experiência de uma turma de medicina. Revista Brasileira de Educação Médica, 2013; 37(3): 448-454.

18. SOUZA MC, et al. Inovação metodológica na integração ensino-serviço na educação médica. Medicina (Ribeirão Preto On line), 2016; 49(3): 284-291.

19. TEMPSK P e BORBA M. O SUS como escola. Revista Brasileira de Educação Médica, 2009; 3(33): 319-320. 